\title{
COMPUTER FILTERING OF IMAGES OF IONOGRAMS OF HF IONOSPHERIC RADIO COMMUNICATION PATHS AND AN ALGORITHM FOR DETERMINING SIGNAL-TO-NOISE RATIO
}

\author{
Vladimir Ivanov* Dmitriy Ivanov Natalya Ryabova Mariia Ryabova* Andrei Chernov Alexey Elsukov \\ ${ }^{1}$ Volga State University of Technology, Yoshkar-Ola, Russia
}

The algorithm of obtaining ionograms of oblique sounding of wideband radio channels was implemented in software. The paper presents an algorithm of computer filtering of ionograms with the use of median equalization. The algorithm of determining signal-to-noise ratio in HF ionospheric radio channel was developed and implemented in software.

Key words: Software, lonosphere, Filters, Channels, Algorithms

\section{INTRODUCTION}

Computer image processing methods have been widely used in various fields. Currently, one of the topical scientific objectives is an image processing aimed at extracting data on the object under study. In particular, it is crucial to extract data on the parameters of multi-dimensional HF ionospheric radio communication channels from the ionogram images obtained by ionospheric sounding [01-03]. However, these images are contaminated with noise and radio frequency interferences (RFI) stem from other users. Furthermore, the useful signal and interference signal are stochastic processes. Thus, it is crucial to filter ionogram image. In this case, the filtered image represents the useful signal. We have assumed that it will allow to apply the algorithms of automatically determining the parameters of HF communication channels.

We shall note that theoretical studies were enhanced with the results obtained in studies of ionogram filtering by various authors [04-06]. In this study we applied computer filtering methods including whitening, median equalization as well as threshold method. The originality of the research is to develop an algorithm that can be automatically applied to an ionogram. The algorithm is aimed at determining signal-to-noise ratio in radio channels of HF range and adequate for choosing an optimum channel.

\section{ALGORITHM OF OBTAINING IONOGRAM}

Let us suppose that sounding is carried out with the use of a continuous signal with linear frequency modulation (LFM) i.e. frequency modulated continuous wave (FMCW):

$$
\begin{aligned}
& u_{T}(t)=\cos \left(\omega_{0} t+\gamma t^{2}\right) \\
& \omega=\omega_{0}+2 \gamma t
\end{aligned}
$$
where $2 \gamma=\frac{d \omega}{d t}=2 \pi \frac{d f}{d t}=2 \pi \dot{f}$ - angular frequency
sweep rate.

Signal complex envelope is as follows:

$$
U_{T}(t)=\exp j \gamma t^{2}
$$

Let us suppose that the propagation of signal in a radio channel excites $M$ discrete modes. Each propagation mode gets an attenuation $s_{m}$, phase delay $\tau_{p m}$ and group delay $\tau_{\text {gm }}$, as well as additive noise which is as follows:

$$
u_{n}(t)=U_{0 n}(t) \exp j v_{n}(t) \cdot \exp j \omega t=u_{0 n}(t) \cdot \exp j \omega t
$$

where $U_{0 n}(t), v_{n}(t)$ - real-valued functions, and $u_{0 n}(t)$ - complex-valued random slowly varying function of time. The received signal is divided into adjacent segments. Each segment is multiplied by the Gaussian type window function. We used Gaussian function.

Amid-band("carrier")frequency is $\omega_{c k}=\omega_{0}+2 \gamma \cdot\left(\bar{t}_{k}-T_{a}\right)$ for each $k$-th segment. Thus, signal phase can be represented as $\omega_{c k} t+\gamma t^{2}$.

The window length (analysis time) $2 T_{a}$ is significantly greater than the delay in a channel $\left(T_{a} \gg \tau_{p m}\right)$, so a complex envelope of a received signal at the carrier $\omega_{c k}$ is as follows:

$$
\begin{aligned}
& U_{R}(t)+u_{0 n}(t)=\sum_{m=1}^{M} s_{m} \exp \left[-\frac{t^{2}}{T_{a}^{2}}\right] \cdot \exp j\left[\gamma\left(t-\tau_{p m}\right)^{2}\right]+ \\
& +U_{0 n}(t) \cdot \exp \left[-\frac{t^{2}}{T_{a}^{2}}\right] \cdot \exp j v_{n}(t)
\end{aligned}
$$

We shall note that the parameters of this function $s_{m}, \tau_{p m}, U_{0 n}$ depend on the segment carrier frequency $\omega_{c k}$. 
Let us consider a module of spectrum of function $\exp \left[-\frac{t^{2}}{T^{2}} \cdot \exp j\left[r^{2}\right]\right.$, which, according to [06], is equal to $\left.\frac{2 \sqrt{\pi} r T_{a}^{2}}{\exp }-\frac{\omega^{2}}{\sigma^{2}}\right]$ where $\omega_{s} \approx 2 \gamma T_{a}$ - is half a bandwidth in terms of angular frequency. Let us estimate bandwidth $B_{s}$ of a compressed signal in terms of angular frequency:

$$
B_{s} \approx \frac{2 \omega_{s}}{2 \pi}=2 \frac{\gamma}{\pi} T_{a}=2 \dot{f} T_{a}
$$

In order to compress segment defined by a Gaussian window in the frequency domain (4), it is multiplied by the function:

$$
\hat{s}(t)=\exp j\left\lfloor-\gamma \cdot t^{2}\right]
$$

Then a compressed signal which is also called difference frequency signal is obtained as a product of functions (4) and (6), and is as follows:

$$
\begin{aligned}
& U_{R d}(t)=\left[U_{R}(t)+u_{0 n}(t)\right] \cdot \hat{s}(t)= \\
& =\sum_{m=1}^{M} s_{m} \exp \left[-\frac{t^{2}}{T_{a}^{2}}\right] \cdot \exp j\left[-2 \gamma \cdot \tau_{p m} \cdot t+\gamma \tau_{p m}^{2}\right]+ \\
& +U_{0 n}(t) \cdot \exp \left[-\frac{t^{2}}{T_{a}^{2}}\right] \cdot \exp j v_{n}(t) \cdot \exp j\left[-\gamma \cdot t^{2}\right]= \\
& =\sum_{m=1}^{M} s_{m} \exp \left[-\frac{t^{2}}{T_{a}^{2}}\right] \cdot \exp j\left[\Omega_{m} t\right]+ \\
& +U_{0 n}(t) \cdot \exp \left[-\frac{t^{2}}{T_{a}^{2}}\right] \cdot \exp j v_{n}(t) \cdot \exp j\left[-\gamma \cdot t^{2}\right]
\end{aligned}
$$

where $\tau_{p m}(\omega)$ - phase delay of a wave in a channel. The equation for difference frequency is as follows:

$$
\begin{aligned}
& \Omega_{m}=\frac{d}{d t}\left[-2 \gamma \cdot \tau_{p m}(\omega) t+\gamma \tau_{p m}^{2}\right]=\frac{\partial}{\partial t}\left[-2 \gamma \cdot \tau_{p m}(\omega) t+\gamma \tau_{p m}^{2}\right]+ \\
& +\frac{d \omega}{d t} \frac{\partial}{\partial \omega}\left[-2 \gamma \cdot \tau_{p m} t+\gamma \tau_{p m}^{2}\right] \approx \\
& \approx-2 \gamma \tau_{p m}-(2 \gamma)^{2}\left[-\tau_{p m} \frac{\partial \tau_{p m}}{\partial \omega}+\frac{\partial \tau_{p m}}{\partial \omega} \cdot t\right]=-2 \gamma \cdot \tau_{g m}
\end{aligned}
$$

where $\tau_{g m}=\tau_{p m}+\omega \frac{\partial \tau_{p m}}{\partial \omega}$ - group delay.

Let us calculate the spectrum of the signal (7) over the analysis time :

$$
\begin{aligned}
& U_{d}(\Omega)=\int_{-T_{a}}^{T_{a}} U_{d}(t) \exp (-j \Omega t) d t \approx \\
& \left.\approx \sum_{m=1}^{M} s_{m} \int_{-\infty}^{\infty} \exp \left[-\frac{t^{2}}{T_{a}^{2}}\right] \cdot \exp j\left[\Omega_{m} t\right] \cdot \exp j[-\Omega t] d t+9\right) \\
& \left.+\int_{-\infty}^{\infty} U_{0 n}(t) \cdot \exp j v_{n}(t) \cdot \exp \left[-\frac{t^{2}}{T_{a}^{2}}\right] \exp \left[-j \gamma \cdot t^{2}\right)\right] \exp j[-\Omega t] d t
\end{aligned}
$$

The integral is indefinite due to the Gaussian function (window function). Thus, the spectrum is as follows (9):

$$
U_{d}(\Omega) \approx \sum_{m=1}^{M} s_{m} \cdot \sqrt{\pi} T_{a} \exp \left[-\frac{\left(\Omega-\Omega_{m}\right)^{2} T_{a}^{2}}{4}\right]+
$$

$\left.+\int_{-\infty}^{\infty} U_{0 n}(t) \cdot \exp j v_{n}(t) \cdot \exp \left[-\frac{t^{2}}{T_{a}^{2}}\right] \exp \left[-j \gamma \cdot t^{2}\right)\right] \exp j[-\Omega t] d t=$

$$
=U_{R d}(\Omega)+U_{n}(\Omega)
$$

Let us consider a random propagation mode of a compressed chirp signal. Its power spectrum is as follows:

$$
S_{R m}(\Omega)=\left|U_{R d m}(\Omega)\right|^{2}=\pi s_{m}^{2} T_{a}^{2} \exp \left[-\frac{\left(\Omega-\Omega_{m}\right)^{2} T_{a}^{2}}{2}\right]
$$

In this case $\Omega$ is a difference frequency. We represent it according to $\Omega_{m}=-2 \gamma \tau_{g m}$ as $\Omega=-2 \gamma \tau$, where $\tau$ is a random delay. Thus, the power spectrum is as follows:

$$
\begin{aligned}
& S_{R m}(\tau)=\pi s_{m}^{2} T_{a}^{2} \exp \left[-2 \gamma^{2}\left(\tau-\tau_{g m}\right)^{2} T_{a}^{2}\right]= \\
& =\pi s_{m}^{2} T_{a}^{2} \exp \left[-\frac{\pi^{2}}{2}\left(\tau-\tau_{g m}\right)^{2} B_{s}^{2}\right]= \\
& =\frac{\pi}{\gamma} s_{m}^{2} \eta \cdot \exp \left(-2 \cdot \frac{\left(\tau-\tau_{g m}\right)^{2}}{\left(T_{a} / \eta\right)^{2}}\right)
\end{aligned}
$$

where $\eta=\gamma \cdot T_{a}^{2}=\frac{\pi}{2} B_{s} T_{a}$ - bandwidth-duration product of a chirp signal segment.

Power of a random propagation mode at the output of a matched filter (after compression in the time domain) is as follows [07]:

$$
\begin{aligned}
& P_{R m}(\tau)=s_{m}^{2} \eta \cdot \exp \left(-2 \cdot \frac{\left(\tau-\tau_{g m}\right)^{2}}{\left(T_{a} / \eta\right)^{2}}\right)=\frac{\gamma}{\pi} \cdot S_{R m}(\tau)= \\
& =\dot{f} \cdot S_{R m}(\tau)=\eta \cdot\left|h_{m}(\tau)\right|^{2}
\end{aligned}
$$

where $h(\tau)=\sum_{m=1}^{M} h_{m}(\tau)$ - channel impulse response.

Sum of functions $\left|h_{m}(\tau)\right|^{2}$ is a power delay profile (PDP) of a channel and expressed in terms of power spectrum using the following equation:

$$
\begin{aligned}
& \operatorname{PDP}\left(\omega_{c k}, \tau\right)=\sum_{m=1}^{M}\left|h_{m}\left(\omega_{c k}, \tau\right)\right|^{2}= \\
& =\frac{1}{\eta} \sum_{m=1}^{M} P_{R m}\left(\omega_{c k}, \tau\right)=\frac{\gamma}{\pi \eta} \cdot \sum_{m=1}^{M} S_{R m}\left(\omega_{c k}, \tau\right)
\end{aligned}
$$

It can be seen that all the functions are directly proportional to a channel PDP. 
Figure 1a, b shows an ionoaram and a PDP of a channel (at a frequency $\frac{\omega_{c k}}{2 \pi}=f_{c k}=11 \mathrm{MHz}$ ), taken on the Cyprus - Yoshkar-Ola path on 25 March 2015 at 13:20 LT. The observed delays $\Lambda=(7-16.5 \mathrm{~ms})$ was selected to ensure analyzed delays range at least twice greater than the range of the delays of the received signal. The peaks on the PDP plot correspond to the delays of the received modes $\tau=\tau_{g m}$, which are tracks on a noise background. It can be seen that the useful signal concentrates around these peaks. Noise is received for the delays where no signal. Figure $1 \mathrm{~b}$ shows that noise was received at 13.5 ms and above.

Each PDP can be considered as a sequence of pixels in a column. A pixel has a size , where and is determined by the exponent in the equation (13) and is equal to . Signal (channel) bandwidth is limited due to the dispersion in the ionosphere and does not exceed $40 \mathrm{kHz}$. Thus, $\delta f \leq 40 \mathrm{kHz}, \delta \tau \geq 25 \mathrm{mcs}, f_{c k}=k \cdot \delta f$, (where $k=1,2 \ldots$ ) and $\tau=p \cdot \delta \tau$ (where $p=1,2 \ldots$ ).

lonogram is a bitmap image which consist of channel PDPs ordered by mid-band frequencies $f_{c k}$. It shows the variation of a PDP with the change in a mid-band frequency. Color indicates signal power level corresponding to a pixel, so one can judge the modes structure and the channel budget. Besides, an ionogram allows to evaluate the maximum usable frequency and the lowest usable frequency.

The problem is that a received signal is mixed with wideband background noise and narrowband interferences which pose a significant challenge for automatic computer processing of ionograms aimed at determining channel parameters.

Figure 2 presents the original ("contaminated") ionogram taken on Cyprus-Yoshkar-Ola radio path on 27 July 2015 at 17:00 LT.

Narrow band interferences cause vertical lines on the ionogram image as well as stochastic noise causes "rippled" background. The aim of computer filtering is to remove background noise and narrowband interferences.

\section{COMPUTER FILTERING OF IONOGRAM IMAGE WITH THE USE OF MEDIAN EQUALIZATION}

It is known [08] that there are a number of methods for computer image filtering. An ionogram is variable in time. Thus, it is crucial to study the effectiveness of various computer filtering methods.

First, we studied the efficiency of the median equalization method. We shall note that this method is well known [09] and is mainly used to remove stochastic noise. The experiments have shown that median filtering of columns provides the best filtration of "horizontally stretched" tracks. In this case, variational series is built for each PDP and the median is determined. Then the PDP samples are divided by the median value. We shall note that when the columns are ordered by frequency (as in this case), the median filtering is equivalent to median equalization.
Figure 3 shows the result of computer filtering of an ionogram shown in Figure 2 with the use of median equalization.

It can be seen that this method can effectively mitigate background noise and narrowband interferences.

It is known that narrow band interferences are removed by whitening equalizer which is used at the receiver input (before matched filter processing). Figure 4 presents the result of whitening equalizer applied for the ionogram shown in Figure 2.

It can be seen that the algorithm removes narrow band interferences, but does not filter stochastic noise. In order to filter stochastic noise and narrow band interferences we applied both whitening and median equalization methods.

Figure 5 presents the result of successive applying both methods. It can be seen that the background noise became more homogeneous. It allows to apply threshold filtering method. An ionogram presented at Figure 6 proves the effectiveness of successive applying of whitening equalization, median equalization and threshold methods. In this case, the threshold is $5 \mathrm{~dB}$. It can be seen that successive applying of several method allows to solve the problem of computer filtering of ionogram image and "split" signal and noise.

\section{ALGORITHM OF DETERMINING SIGNAL-TO-NOISE RATIO IN HF COMMUNICATION CHANNELS}

A sounding signal is a physical model of a communications signal, so experimental data obtained after sounding can be used in a communication system. Splitting of signal and noise by means of computer filtering allows to estimate signal-to-noise ratio (SNR), which is a key parameter of any communication system. In order to verify the algorithm, let us consider the Equation (10) and noise conversion. Let us calculate the Fourier transform of two product terms in function :

$$
\begin{aligned}
& u_{n}(\Omega)=\int_{-\infty}^{\infty} u_{n}(t) \cdot \exp j v_{n}(t) \cdot \exp j[-\Omega t] d t \text { - noise spectrum, } \\
& H_{f}(\Omega)=\int_{-\infty}^{\infty} \exp \left[-\frac{t^{2}}{T_{a}^{2}}\right] \cdot \exp \left[-j \gamma \cdot t^{2}\right] \exp j[-\Omega t] d t= \\
& =\int_{-\infty}^{\infty} h_{f}(t) \cdot \exp j[-\Omega t] d t \text { - filter frequency response }
\end{aligned}
$$

The equation (15b) takes into account that the noise goes through the filter, which has an impulse response is as follows:

$$
h_{f}(t)=\exp \left[-\frac{t^{2}}{T_{a}^{2}}\right] \exp j\left[-\gamma \cdot t^{2}\right]
$$




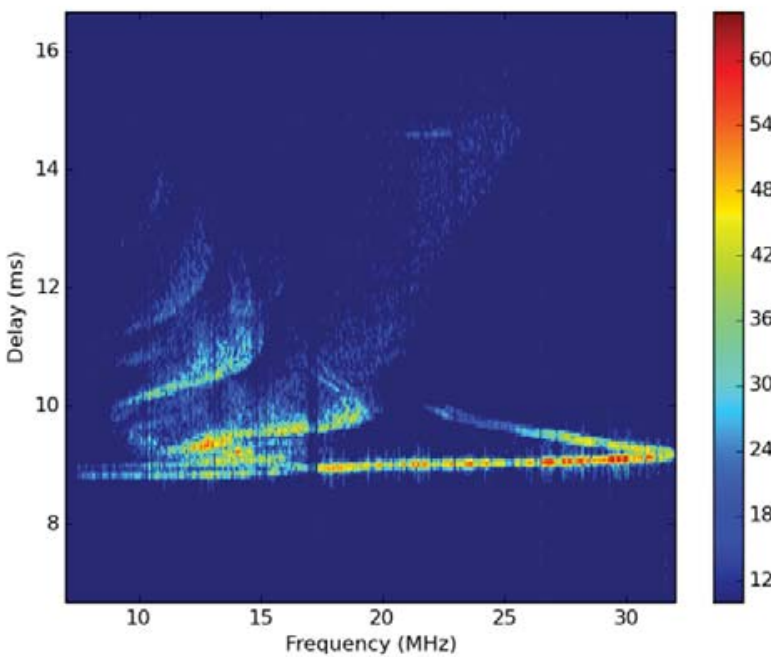

15a)

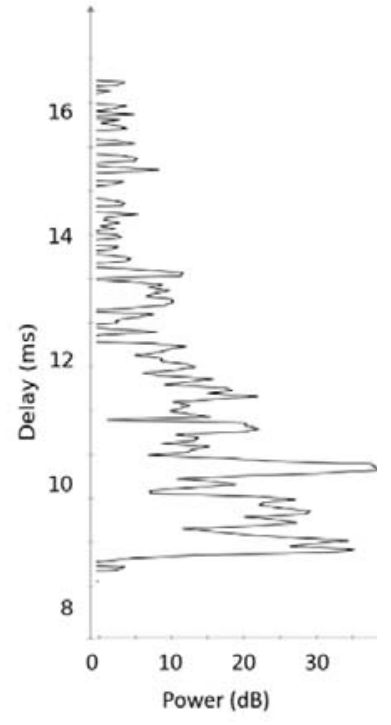

15b)

Figure 1: lonogram - (a) and a PDP of a channel with a bandwidth of $40 \mathrm{kHz}$ at a mid-band frequency of $11 \mathrm{MHz}-(b)$

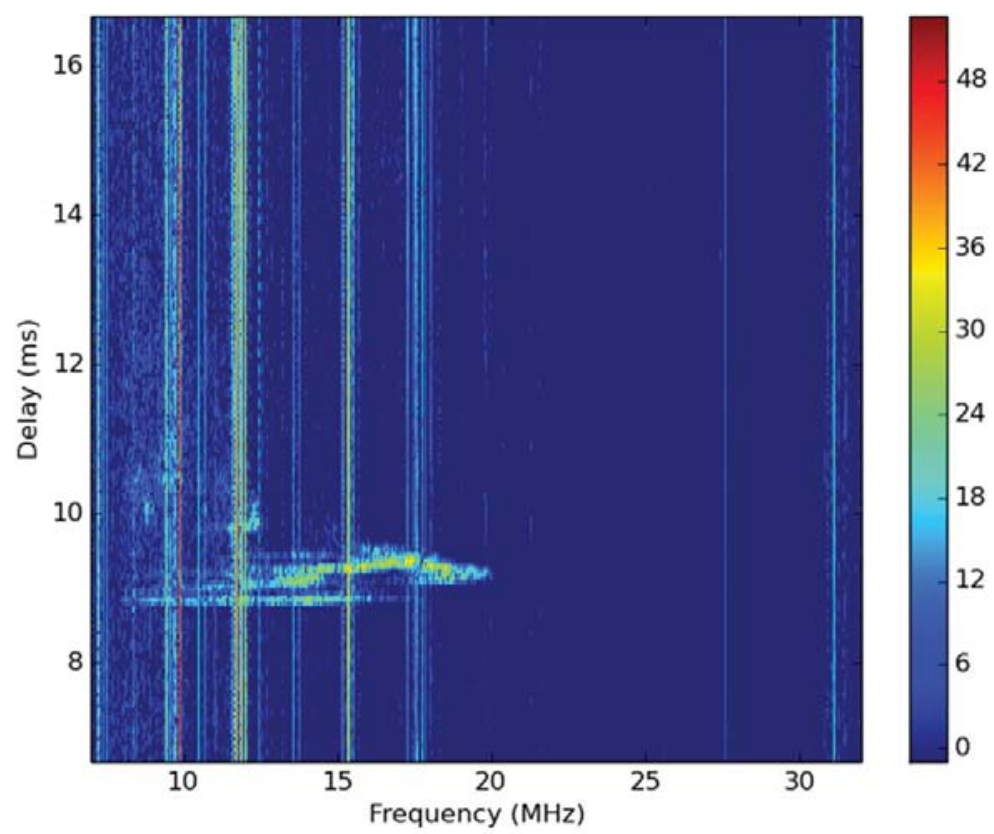

Figure 2: The original ionogram image taken on Cyprus - Yoshkar-Ola path on 27 July 2015 at 17:00 LT 


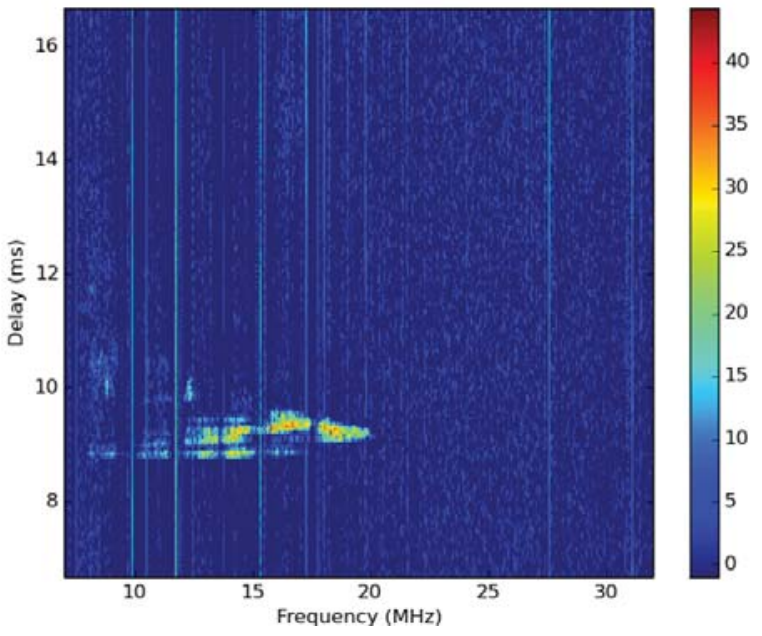

Figure 3: The result of median equalization of an ionogram image

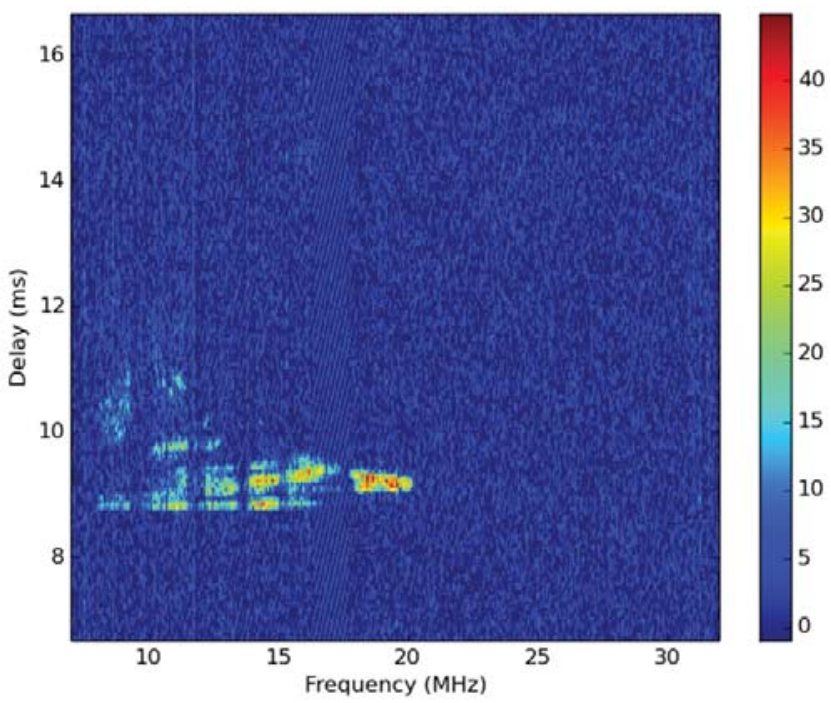

Figure 5: The result of applying two successive methods (whitening and median equalization)

For such filter and $\gamma T_{a}^{2} \gg 1$ the frequency response is as follows [09]:

$$
H_{f}(\omega)=\frac{\sqrt{\pi \gamma T_{a}^{2}}}{\Omega_{f}} \exp \left[-\frac{\omega^{2}}{\Omega_{f}^{2}}\right] \exp j\left[\gamma T_{a}^{2} \frac{\omega^{2}}{\Omega_{f}^{2}}-\frac{\pi}{4}\right]
$$

where $\Omega_{f}=2 \gamma T_{a}$ - half a filter bandwidth for $e^{-1}$ level. The square of a modulus of a filter frequency response is as follows:

$$
\left|H_{f}(\Omega)\right|^{2}=\frac{\pi \gamma T_{a}^{2}}{\Omega_{f}^{2}} \exp \left[-\frac{2 \Omega^{2}}{\Omega_{f}^{2}}\right]
$$

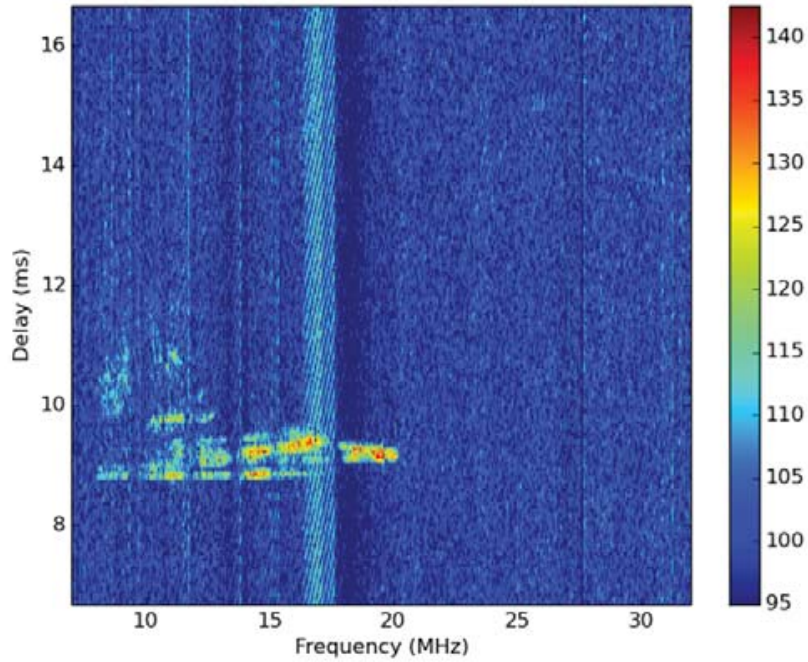

Figure 4: The result of applying whitening equalizer

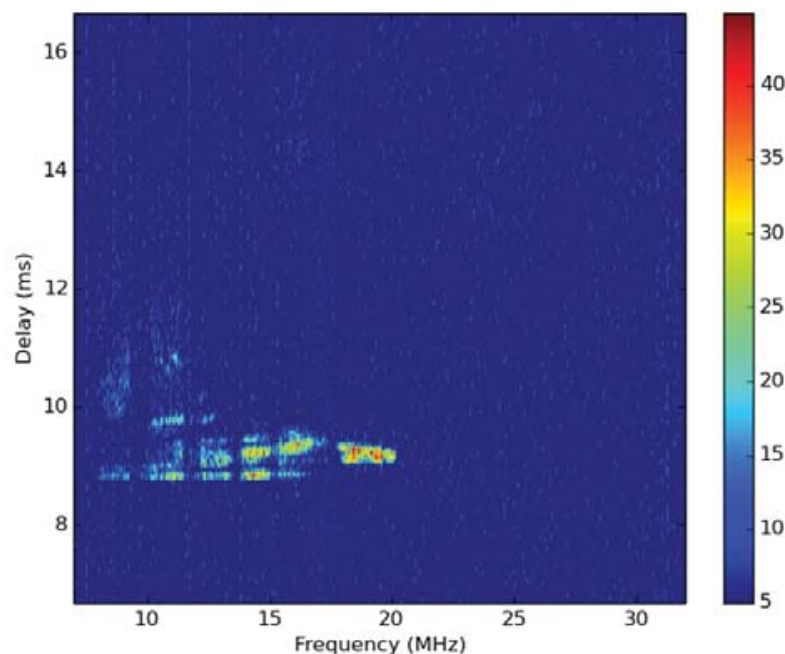

Figure 6: The result of successive applying of whitening equalization, median equalization and threshold methods

Noise power spectral density at the filter output can be obtained by multiplying the square of the frequency response to the power spectral density at the filter input [10]:

$$
\begin{aligned}
& N_{\text {out }}(\Omega)=\left|H_{f}(\Omega)\right|^{2} N_{\text {in }}(\Omega)= \\
& =\frac{\pi \gamma T_{a}^{2}}{\Omega_{f}^{2}} \exp \left[-\frac{2 \Omega^{2}}{\Omega_{f}^{2}}\right] N_{\text {in }}(\Omega)= \\
& =\frac{\pi \gamma T_{a}^{2}}{\Omega_{f}^{2}} \frac{n_{0}}{2} \exp \left[-\frac{2 \Omega^{2}}{\Omega_{f}^{2}}\right]
\end{aligned}
$$

Let us consider a random propagation mode of a compressed chirp signal. Its power spectral density is as follows:

$\left.W_{R m}(\Omega)=\frac{\left|U_{R d m}(\Omega)\right|^{2}}{2 T_{a}}=\frac{\pi}{2} s_{m}^{2} T_{a} \exp \left[-\frac{\left(\Omega-\Omega_{m}\right)^{2} T_{a}^{2}}{2}\right] 19\right)$ 
The maximum value of the spectral density is as follows:

$$
\max \left\{W_{R m}(\Omega)\right\}=W_{R m}(0)=\frac{\pi}{2} s_{m}^{2} T_{a}
$$

Mode energy is as follows:

$$
\begin{aligned}
& E_{R m}=\int_{-\infty}^{\infty}\left|U_{R d m}(t)\right|^{2} d t=\int_{-\infty}^{\infty}\left|U_{R d m}(\omega)\right|^{2} d \omega= \\
& =s_{m}^{2} \frac{T_{a}}{\sqrt{2}} \int_{-\infty}^{\infty} \exp \left(-u^{2}\right) d u=s_{m}^{2} \sqrt{\frac{\pi}{2}} T_{a}= \\
& =\sqrt{\frac{2}{\pi}} \max \left\{W_{R m}(0)\right\} \approx 0,8 \cdot \max \left\{W_{R m}(0)\right\}
\end{aligned}
$$

We have assumed that in this case SNR for a random propagation mode could be estimated as follows:

$$
S N R_{m}=\frac{W(0)}{\bar{N}_{\text {out }}(\Omega)}=\frac{2 s_{m}^{2} T_{a}}{n_{0}}
$$

We have assumed that the is the ratio of the average signal power to average noise power. In this case, the average power of a Gaussian pulse with duration $2 T_{a}$ equals to:

$$
P_{c p m} \approx \frac{1}{2 T_{a}} \int_{-\infty}^{\infty}\left|U_{R d m}(t)\right|^{2} d t=\frac{1}{2} \sqrt{\frac{\pi}{2}} s_{m}^{2}
$$

So the energy of a received random mode of a difference frequency signal in a Gaussian window equals to:

$$
E_{m} \approx P_{m c p} \cdot 2 T_{a}
$$

Thus, the equation for the signal-to-noise ratio is as follows:

$$
S N R_{m}=\sqrt{\frac{2}{\pi}} \cdot \frac{2 E_{m}}{n_{0}}=0,8 \frac{2 E_{m}}{n_{0}}
$$

Since the total energy of the received signal is the sum of the energies of different modes $E=\sum^{M} E_{m}$, the signal to-noise ratio could be estimated as follows:

$$
S N R=\sum_{m=1}^{M} S N R_{m}=\sqrt{\frac{2}{\pi}} \cdot \frac{2 \sum E_{m}}{n_{0}}=0,8 \frac{2 E}{n_{0}}
$$

We shall note that if the mode power is $10 \mathrm{~dB}$ less than the global maximum, its contribution can be neglected [07].

Let us consider the algorithm of determining SNR in the case when the signal processing includes equalization methods which divide signal + noise (SN) samples at a certain level of noise $\bar{N}$.

In the case of the median equalization samples are divided by the median value $\left(M_{e}\right)$. We shall note that each sample represents the average power which corresponds to the spectrum analyzer resolution. It can be either the noise power $P_{n} \cdot \delta F$, or SN power $\left(P_{s}+P_{n}\right) \cdot \delta F$ . Let us prove that $P_{n}=k \cdot M_{e}(N)$. Coefficient $k$ allows to recalculate the noise median value in its average power. As a result, the following relation is obtained:

$$
\eta=\frac{\left(P_{s}+P_{n}\right) \delta F}{P_{n} \delta F}=\frac{P_{s}}{P_{n}}+1
$$

So:

$$
\begin{aligned}
& S N R=10 \lg \left[\frac{\left(P_{s}+P_{n}\right)}{P_{n}}-1\right]= \\
& =10 \lg \left[\frac{\left(P_{s}+P_{n}\right) \delta F-k \cdot M_{e}(N) \delta F}{k \cdot M_{e}(N) \delta F}\right]
\end{aligned}
$$

Let us estimate the coefficient $k$. We shall note that $P_{n}=U_{0 n}^{2}=y$. Noise amplitude is $x=U_{0 n}$. We have assumed that the noise is a quasi-white, i.e. its spectral density is constant in a chirp signal bandwidth $[-\pi B, \pi B]$. In this case, the noise amplitude is distributed according to the Rayleigh law with parameter $\sigma$ :

$$
p(x)=\frac{x}{\sigma^{2}} \exp \left(-\frac{x^{2}}{2 \sigma^{2}}\right)=\frac{x}{\sigma^{2}} e^{-\frac{x^{2}}{2 \sigma^{2}}}
$$

The distribution for the value could be obtained by functional transformation $x=\sqrt{y}$.

As a result, the density distribution of is as follows:

$$
g(y)=\frac{1}{2 \sigma^{2}} \exp \left(-\frac{y}{2 \sigma^{2}}\right)
$$

The median value could be calculated using the following equation:

$$
\int_{a}^{M_{e}} \frac{1}{2 \sigma^{2}} \exp \left(-\frac{y}{2 \sigma^{2}}\right) d y=0,5
$$

So:

$$
M_{e}=2 \ln 2 \cdot \sigma^{2}
$$

It is clear that the average noise power is equal to:

$$
\bar{P}_{n}=M_{1}[y]=2 \sigma^{2}
$$

Thus, coefficient $k$ could be obtained using the following equation:

$$
k=\frac{\bar{P}_{n}}{M_{e}}=\frac{1}{\ln 2} \approx 1,4
$$

Figure 7 presents is an algorithm of determining frequency dependence of the signal-to-noise ratio in different frequency radio channels. 


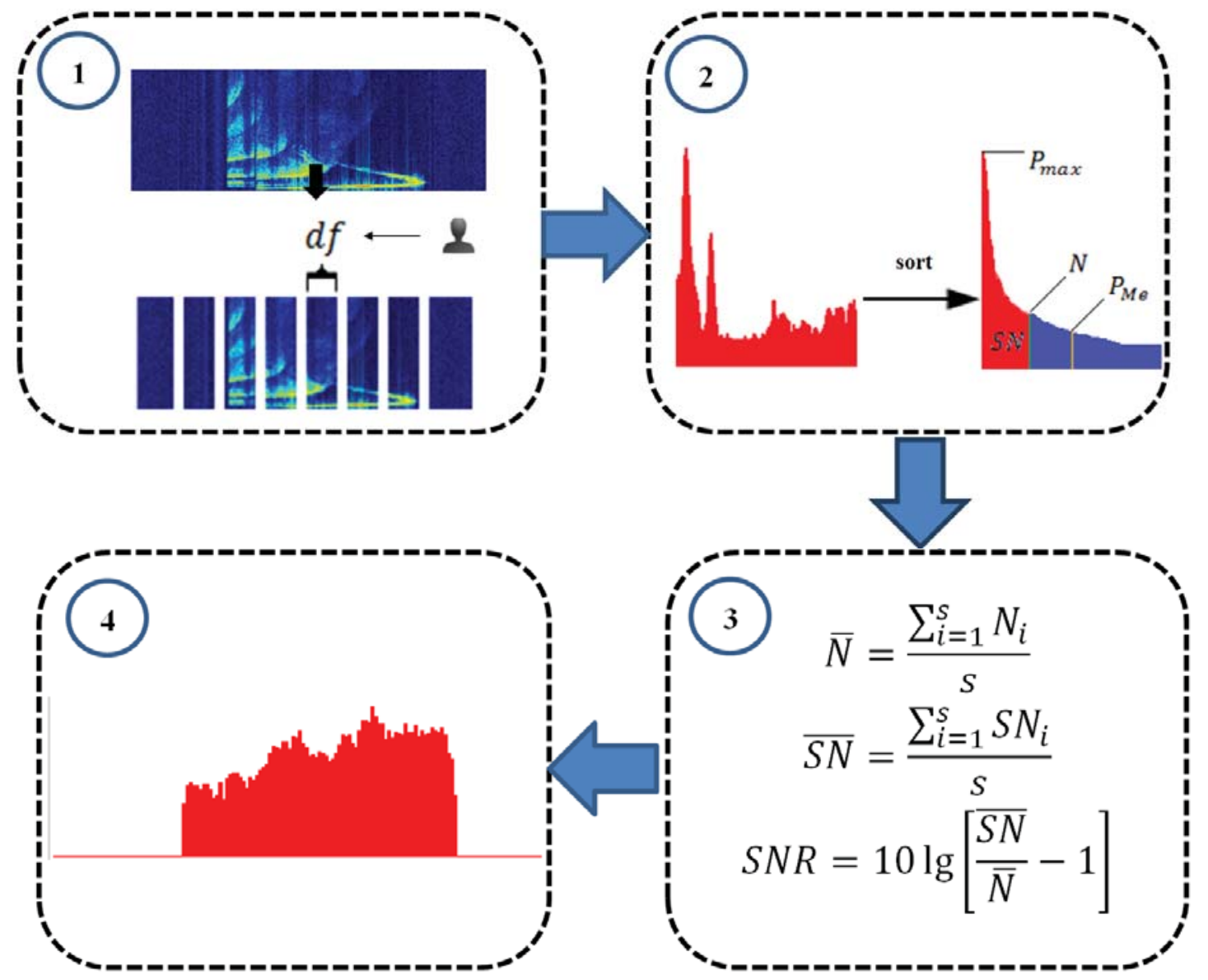

Figure 7: The algorithm of determining signal-to-noise ratio with the use of computer filtering of ionogram image

The algorithm consists of the following steps:

1. Split frequency band into intervals of length $d f$ with $s=\frac{m \cdot d f}{f_{k}-f_{r}}$ PDPs in each interval.

2. For each PDP:

- calculate median value of the received signal power $P_{M e}$;

- calculate the noise power level as $N=1,4 \cdot P_{M e}$;

- calculate the maximum signal power $P_{\max }$;

- calculate the sum SN of selected samples.

3. Calculate for all PDPs in the interval :

- average noise power level $\bar{N}=\frac{\sum_{i=1}^{s} N_{i}}{s}$ :

- average level of signal-noise mixture $\bar{S} \bar{N}=\frac{\sum_{i=1}^{s} S N_{i}}{S}$;

- calculate signal-to-noise ratio $S N R=10 \lg \left[\frac{\bar{S} \bar{N}}{\bar{N}}-1\right]$.

4. Plot a histogram with column width and height SNR.
Figure 8 shows the estimates of frequency dependence of SNR for the following intervals $d f=40 ; 200 ; 400 \mathrm{kHz}$ as well as unequalized and equalized ionograms obtained by sounding Cyprus - Yoshkar-Ola path on 13 March 2015 at 16:23 UT.

It is clear that the SNR estimation stability increases with the increase in the averaging interval.

\section{CONCLUSIONS}

The algorithm of obtaining ionograms of oblique sounding of wideband radio channels was implemented in software. The methods of computer filtering of ionograms images were experimentally verified. The successive applying of whitening equalization, median equalization and threshold methods can effectively remove background noise and narrowband interferences. The algorithm of determining signal-to-noise ratio in HF ionospheric radio channel was developed and implemented in software. 

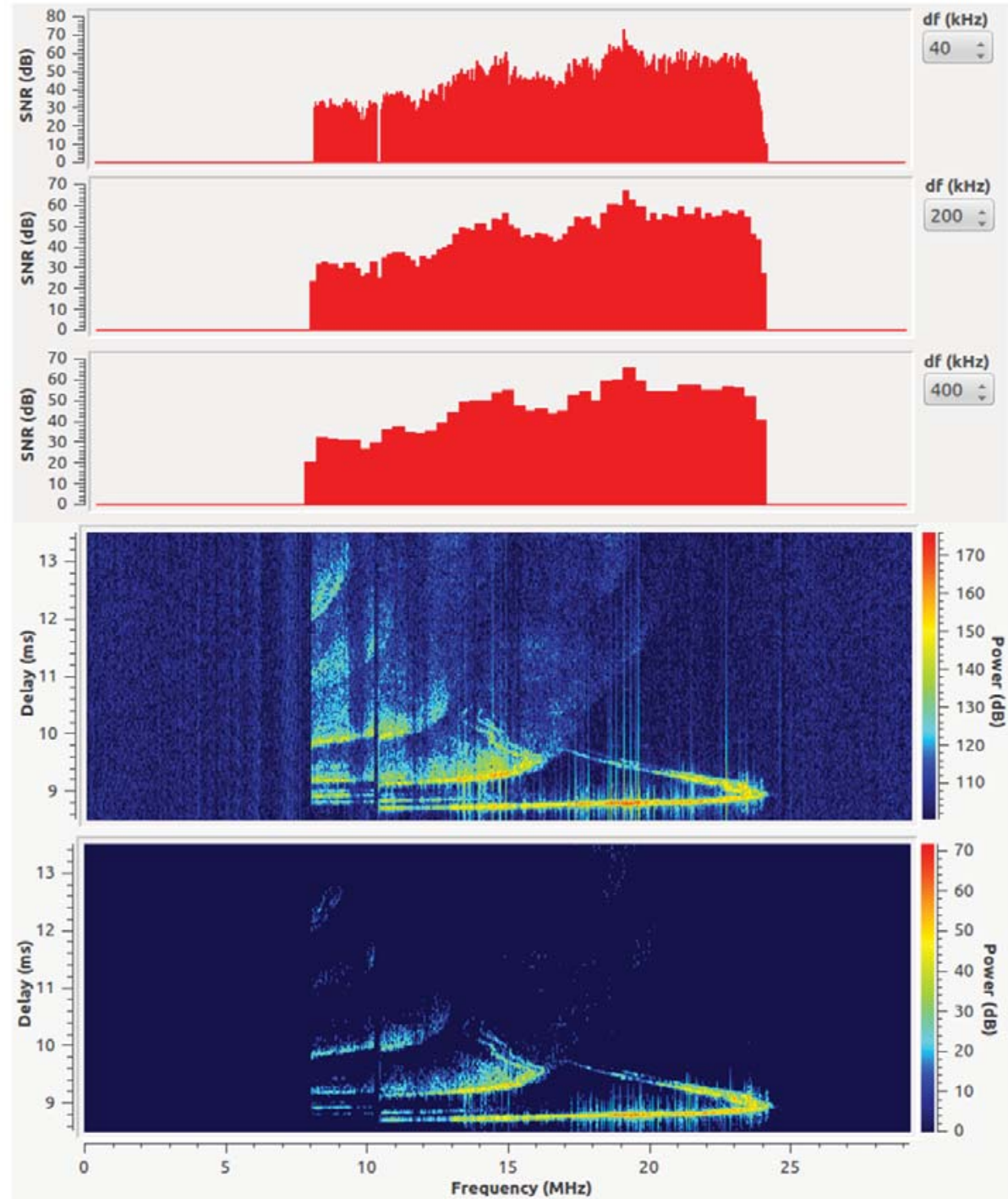

Figure 8: SNR estimated for different intervals $d f=40 ; 200 ; 400 \mathrm{kHz}$ and ionograms taken on Cyprus - Yoshkar-Ola path on 13 March 2015 at 16:23 UT

This work was supported by the grants № 13-07-00371; 13-02-00524; 15-07-05280; 15-07-05294 from the Russian Foundation for Basic Research;

the grants № 3.2695.2014 / K, № 8.2697.2014 / K, № 2276, № 2247 from the Ministry of Education of the Russian Federation;

the grant № 15-19-10053 from Russian Science Foundation.

\section{REFERENCES}

1. Grozov, V.P. The techniques for processing and interpretation of ionosphere sounding data using the continuous chirp signal / V.P. Grozov, V.I. Kurkin, S.N. Ponomarchuk // Physical Bases of Instrumentation. -2012. -V. 1, № 3 (4). - Pp. 33-41.

2. Maslin, N.M. HF communications: a systems approach / N.M. Maslin. - London.: Pitman Publishing, 1987. - P. 89

3. Kryukovsky, A.S. Research of singularities of short radio wave propagation in non-uniform anisotropic ionosphere / A. S. Kryukovsky, D. S. Lukin, D. V. Rastyagaev // Electromagnetic Waves and Electronic Systems. -2009. -V. 14, № 8. -Pp. 17-26. 
4. Ivanov, V.A. Sounding of the ionosphere and decametric communication channels by complex radio signals N.A. Ivanov, D.V. Ivanov, N.V. Ryabova // Vestnik of Volga State University of Technology. Series "Radio Engineering and Infocommunication Systems". -2010. -№ 1. -Pp. 3-37

5. Ivanov, V.A. Comprehensive methods of vertically-oblique sounding ionograms processing for determining parameters of ionospheric channels / V.A. Ivanov, N.V. Ryabova, A.V. Maltcev etc. // Telecommunications and Radio Engineering. 2014. № 8. Pp. 11-21.

6. Ivanov, D.V. SDR-ionosonde with continuous LFM-signal on the platform USRP / D.V. Ivanov, V.A. Ivanov, N.V. Ryabova, A.A. Elsukov, M.I. Ryabova, A.A. Chernov // Vestnik of Volga State University of Technology. Series "Radio Engineering and Infocommunication Systems". 2013. № 3 (19). Pp. 80-93.
7. Ivanov, V.A. Automatic determination of the signalto-noise ratio when measuring the scattering function of narrow-band decameter radio channels / V.A. Ivanov, N.V. Ryabova, I.E. Tsarev, A.V. Korkin // Vestnik of Volga State University of Technology. Series "Radio Engineering and Infocommunication Systems”. 2009. № 3. Pp. 3-12.

8. Cannon, P.S. DAMSON HF channel characterization - a review / P.S. Cannon, M.J. Angling, N.C. Davies et al // Proc. MILCOM. - 2000. - Vol. 1. - Pp. 59-64.

9. Le Roux, Y. M. HF channel modeling and simulation / Y. M. Le Roux, M. Niberon, R. Fleury et al // IEE Radio Receivers and Associated Systems 5th conf. - Cambridge, 1990. - Pp. 72-76.

10. Erukhimov, L.M. Pedersen mode ducting in a randomly stratified ionosphere / L.M. Erukhimov, V.P. Uryadov, Yu.N. Cherkashin, V.A. Eremenko, V.A. Ivanov, N.V. Ryabova, V.V. Shumaev // Waves in Random Media. - 1997. - T. 7, № 4. - C. 531-544. 